\title{
Urban park lighting as a source of botanical light pollution
}

\author{
Robert Supronowicz, Irena Fryc \\ Bialystok University of Technology, Faculty of Electrical Engineering, Wiejska 45a, 15-351 Bialystok
}

Received September 17, 2019; accepted September 30, 2019; published September 30, 2019

\begin{abstract}
That paper describes the relative impact of an artificial lighting device on botanical light pollution, considering spectral power distribution (SPD in the lighting area. This impact is described by the Relative-to-Moon Photosynthesis Index (RMPI) and Induced Phytochrome Index (IPr). We found that in the case when lighting is realized by using LED luminaires instead of high-pressure sodium (HPS) or metal halide (MH) lamps, the influence of spectral light on plant vegetation process amplifies. Additionally, our research shows that estimating botanical light pollution on the basis of lamps' CCT is not the best method and that using SPD is better for this purpose.
\end{abstract}

Nowadays, illuminating green areas, e.g. urban parks, plays an important role in city lighting. By correctly choosing and mounting luminaires with a proper intensity distribution curve it is possible to obtain well-lighted green spaces. Outdoor lighting is mainly realised with downwards and upwards luminaires. Up-lights are special kind of outdoor luminaires mounted mainly in the ground or on facades, and their light distribution provides emission of the entire luminous flux upwards. Their purpose is to light up architecture, facades, walls covered with greenery, trees. The placement of luminaires on the above illuminated surfaces i.e. poles, masts, walls, tops of banner ads, etc. ensures high energy efficiency for lighting installations, but even such scenario does not eliminate the phenomena of light pollution.

A lot has been said about light pollution over the last few decades [1-4]. Such a phenomenon limits or makes impossible astronomic observations and researches from an area with high light pollution. It has a negative influence on flora and fauna, which normally function in absolute darkness [5]. The research shows that an excessive amount of sky glow and light pollution impairs the sleep of people living in cities [6] and is also a source of botanical pollution $[1,5]$.

Contemporary LED lighting devices can be developed in almost any spectral power distribution characteristics. That has made them very popular for scientific and practical applications. In-ground LED luminaires with available color mixing are gaining popularity (RGBTW red, green, blue, tunable white LEDs), while advanced control systems like DALI or DMX allow smooth illumination and preparation of advanced scenario design [7].

*E-mail: r.supronowicz@ doktoranci.pb.edu.pl
In this paper we are focusing on how the spectral power distribution (SPD) of a light source affects botanical light pollution in the lighting area. For this purpose we introduced the Relative-to-Moon Photosynthesis Index RMPI and Induced Phytochrome Index (IPr). The spectral power distribution of light created at full moon (Fig. 1) is used) as a reference light source. The exposure time and levels of illuminance and its uniformity were not included in this study.

Contemporary urban park lighting is realized by using high pressure sodium (HPS) and metal halide (MH) lamps, as well as LED luminaires with different correlated colour temperatures (CCTs) referred to as warm, neutral and cold white. The examples of spectral power distribution of some of those lamps are shown in Fig. 2.

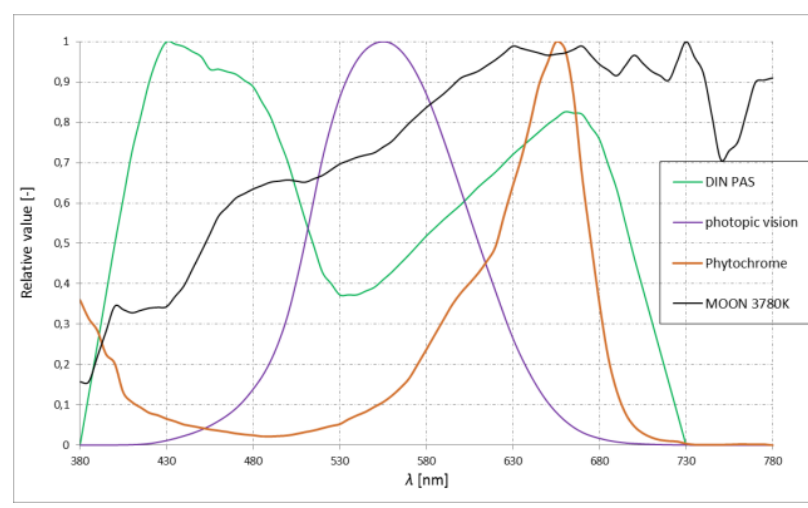

Fig. 1. The spectral responsivity of DIN PAS, phytochrome, photopic $\mathrm{V}(\lambda)$ and SPD of light at full moon.

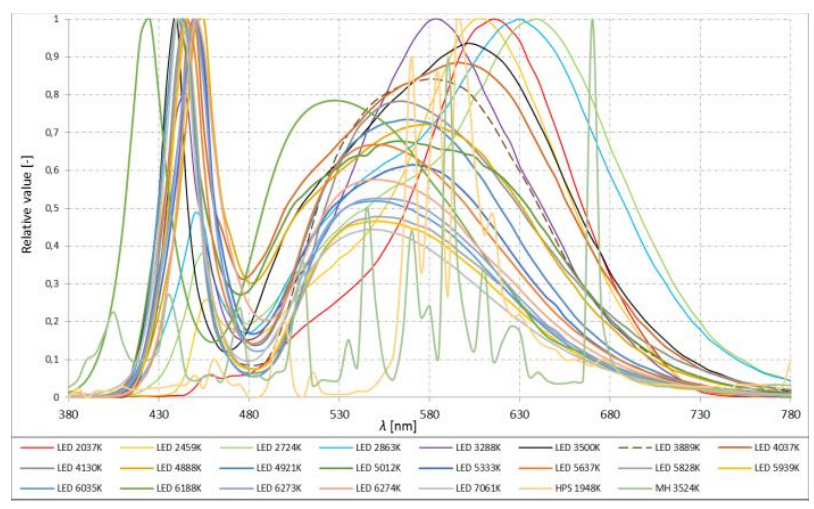

Fig. 2. The SPDs and CCT value of light sources under consideration. 
The RMPI and IPr indexes are described by Eq. (1) and Eq. (2), respectively. For those kinds of calculation, it is important to use lamps which provide equal luminous flux. The SPD of lamps which have equal luminous flux is presented in Fig. 3 and Fig. 4.

$$
R M P I=\frac{\int_{380}^{780} P_{n}(\lambda) \cdot P A S(\lambda) d \lambda}{\int_{380}^{780} P_{M}(\lambda) \cdot P A S(\lambda) d \lambda}
$$

In Eq. (1), $P_{\mathrm{n}}(\lambda)$ is the SPD of a light source, $P_{\mathrm{M}}(\lambda)$ denotes the SPD of light at full moon and $P A S(\lambda)$ describes the photosynthesis action spectrum.

$$
I P r=\frac{\int_{380}^{780} P_{n}(\lambda) \cdot P_{\mathrm{R}}(\lambda) d \lambda}{\int_{380}^{780} P_{M}(\lambda) \cdot P_{\mathrm{R}}(\lambda) d \lambda}
$$

In Eq. (2), $P_{\mathrm{n}}(\lambda)$ is the SPD of a light source, $P_{\mathrm{M}}(\lambda)$ denotes the SPD of light given by a full moon and the $P_{\mathrm{R}}(\lambda)$ is describing phytochrome action spectrum.

Higher values of IPr and RMPI lead potentially to an increased impact on plants.

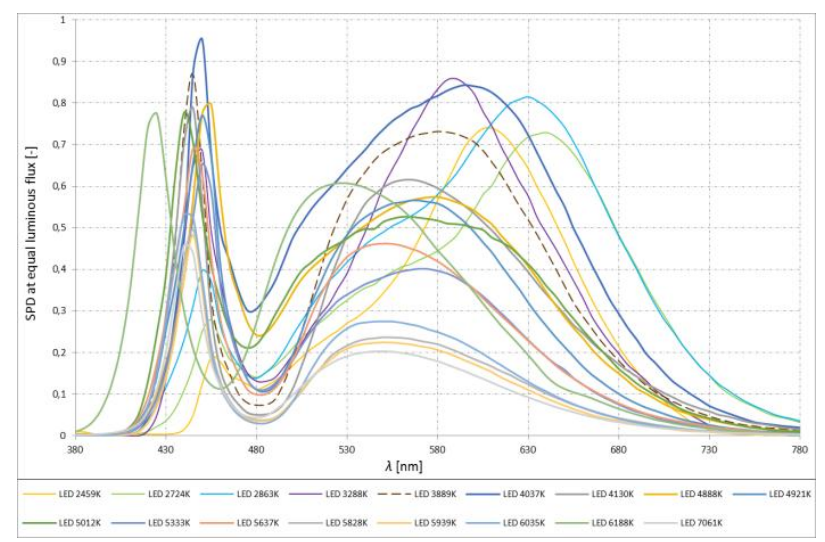

Fig. 3. The SPDs of lamps normalized for their equal luminous flux.

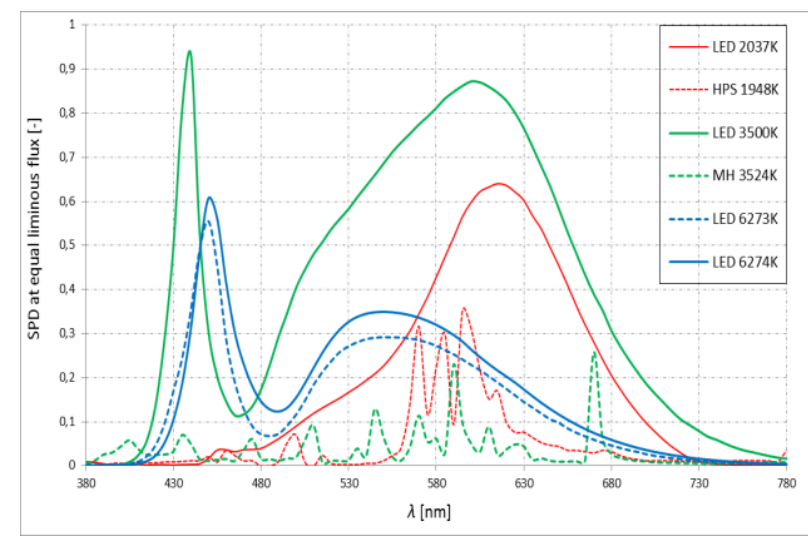

Fig. 4. The SPDs of lamps with similar CCT and normalized for their equal luminous flux.
For RMPI calculations it is also important to know the photosynthesis action spectrum (PAS) of a plant [8] and the $P_{\mathrm{R}}(\lambda)$ [9] phytochrome action spectrum (Fig. 1). The PAS function for each plant varies [10], but the German Institute for Standardization has specified in DIN norms 5031-10 [11] the averaged PAS for plants.

As we can see in Fig. 1, the wavelength range for photopic vision and PAS coincide, so it is impossible to construct an outdoor luminaire which will illuminate an urban park and remain neutral for the vegetation process of flora. It is only possible to limit its impact by choosing the SPD of luminaires.

In Fig. 5 and Fig. 6 there are shown calculated values of RMPI and IPr indices. The data presented in these drawings indicate that there is a relationship between the spectral distribution of light sources and their impact on plants photosynthesis and photomorphogenesis. These issues have been known for a long time, but modern dynamic development of LED technology requires consideration of the impact of LED SPDs on botanical light pollution.

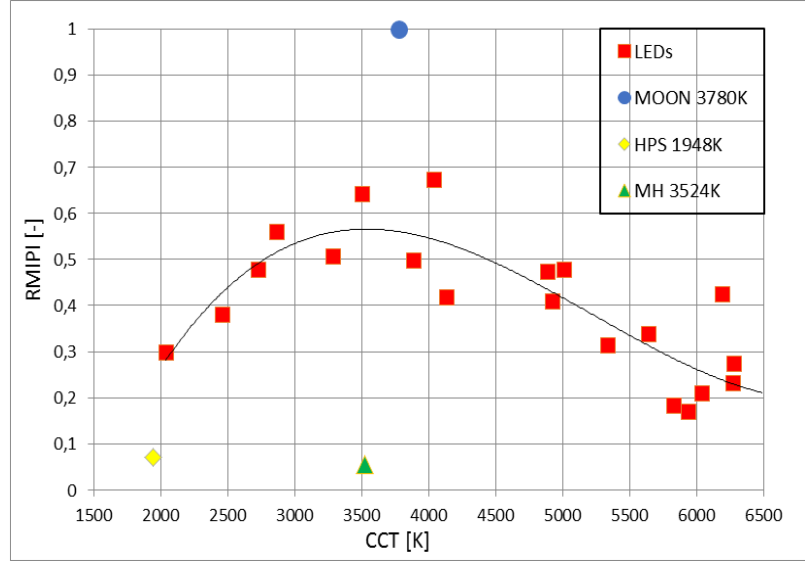

Fig. 5. The RMPI values for LEDs and typical MH and HPS lamps.

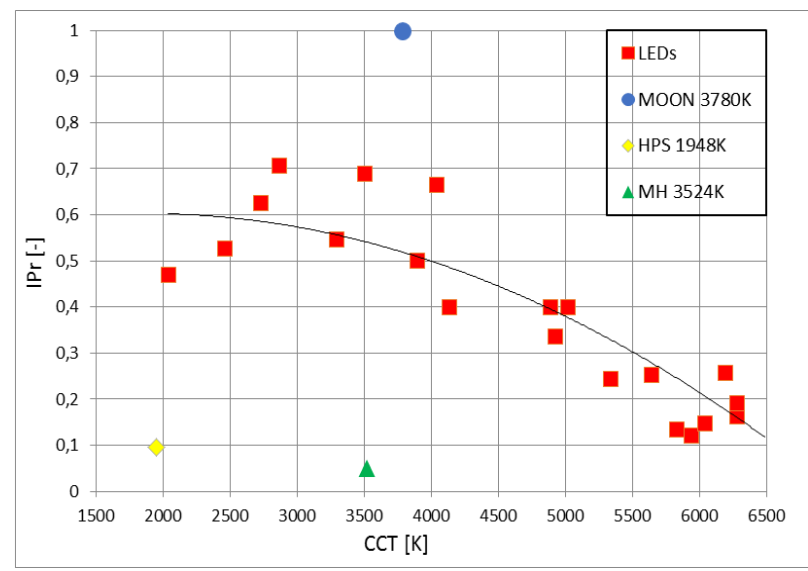

Fig. 6. The IPr values for LEDs and typical MH and HPS lamps. 
As we can see in Fig. 5 and Fig. 6, the RMPI and IPr factor reaches the highest value for LED sources and not $\mathrm{MH}$ and HPS lamps

The RMPI for LEDs is characterized by lower values at warm white and cool white than in the neutral white CCT range. In the cool white range, there are two investigated LEDs with a similar CCT, the RMPI of which differs almost twice, which indicates high dependency on SPD.

And the IPr indicator for LEDs drops by increasing the CCT value. Both indexes for all investigated LED sources do not achieve three fourths of the moon light value.

The value of RMPI and IPr for light at full moon is 1 . It means that the SPD of all investigated LEDs influences less the plants vegetation process than the spectral power distribution of light created by a full moon.

In studying botanical light pollution, we must keep it in mind that illuminance levels created by artificial lighting often are much higher than those given by a full moon. Therefore, the total impact on the vegetation process can be much higher for artificial lighting than for the full moon light, because the exposure time is an additional feature affecting photosynthesis [12].

As a continuation, the authors would like to expand their research on white light created by mixing RGBW (red, green, blue, white) LEDs. This kind of lamps is becoming more and more popular in lighting projects those days and for this reason it should be taken under consideration.

The best option used in outdoor lighting projects would be for the software to contain lighting indicators of botanical light pollution. The indexes like RMPI, IPr, induced photosynthesis index (IPI), melatonin suppression index (MSI) and star light index (SLI) should be accounted for as they would easily indicate how the SPD of used luminaires affects plant vegetation, human circadian rhythm and light pollution, proving some important information for the lighting project.

The authors recommend introducing mandatory standards for outdoor lighting fixtures, especially for areas often visited by people, containing a lot of greenery like urban parks.

The authors suggest that for a very precise analysis of radiation impact on PAS for a specific plant type, it is worth using exactly the PAS of the species [13].

It is interesting to compare HPS, MH, and LED lighting influence on RMPI and IPr index values (Fig. 5 and Fig. 6 ). The calculated values for the tested number of LEDs inform us that the impact of LED luminaires with similar CCTs, as compared to conventional lighting sources, is at least 3 times and greater on photosynthesis and phytochrome action spectrums.

Figures 5 and 6 show that the luminaire with a specified similar CCT has a different impact on vegetation. Those values suggest getting back to less efficient and lower light quality solutions which are $\mathrm{MH}$ and HPS. Some studies suggest as a solution returning to mercury lamps [14], which is irrational from the point of environmental and human needs for efficient and high colorimetric lighting.

This work was supported by the Ministry of Science and Higher Education in Poland under the program number $\mathrm{MB} / \mathrm{WE} / 6 / 2018$.

\section{References}

[1] P. Tabaka, I. Fryc, Landscape Lighting as a Source of Light Pollutionthe Effect of the Seasons on this Phenomenon, Lighting Conference of the Visegrad Countries, Lumen V4, 10.1109/LUMENV.2016.7745541 (IEEE 2016).

[2] I. Fryc, F. Bisegna, P. Tabaka, Environment and Electrical Engineering and 2017 IEEE Industrial and Commercial Power Systems Europe (EEEIC/I\&CPS Europe 2017) IEEE International Conference; doi: 10.1109/EEEIC.2017.7977724.

[3] I. Fryc, P. Tabaka, Przeglad Elektrotechniczny R.93(6), 46 (2017).

[4] H.E. Schwarz et al., Light Pollution: The Global View (ISBN 978-94017-0125-9, 2003).

[5] M. Aube, J. Roby, M. Kocifaj, PLoS ONE 8(7), e67798 (2013); doi:10.1371/journal.pone.0067798.

[6] M.S. Sankhla, K. Sharma, R. Kumar, Galore Inter. J. Appl. Sci. Humanities 3(2), 55 (2019).

[7] M. Tanaka, T. Horiuchi, S. Tominaga, Proc. SPIE 7866, 78660W (2011).

[8] M. Mottus at el., Photosynthetically Active Radiation: Measurement and Modeling (Encyclopedia of Sustainability Science and Technology, 2011).

[9] J. Li, G. Li, H. Wang, X.W. Deng, The Arabidopsis Book 9, e0148 (2011).

[10] J.B. Clark, G.R. Lister, Plant Physiol. 55(2), NCBI 401-6 (1975).

[11] DIN 5031-10, Optical radiation physics and illuminating engineering Part 10: Photobiologically effective radiation, quantities, symbols and action spectra (2018)

[12] C. ChiLing et al., J. Taiwan Agricultural Research 58(2), 146 (2009).

[13] J. Bennie et al., J. Ecology 104(3), 611 (2016); doi: 10.1111/13652745.12551

[14] W.R. Chaney, Does Night Lighting Harm Trees? (Circular FNR-FAQ17, 2002) 